\title{
Surface activity of magnesium during GaN molecular beam epitaxial growth
}

\author{
V. Ramachandran ${ }^{1}$, R. M. Feenstra ${ }^{1}$, J. E. Northrup ${ }^{2}$ and D. W. Greve ${ }^{3}$ \\ ${ }^{1}$ Department of Physics, Carnegie Mellon University, Pittsburgh, Pennsylvania 15213 \\ ${ }^{2}$ Xerox Palo Alto Research Center, 3333 Coyote Hill Road, Palo Alto, California 94304 \\ ${ }^{3}$ Department of Electrical and Computer Engineering, Carnegie Mellon University, \\ Pittsburgh, Pennsylvania 15213
}

\begin{abstract}
Exposure of wurtzite $\mathrm{GaN}$ films grown on $\mathrm{Si}$-polar $6 \mathrm{H}-\mathrm{SiC}(0001)$ to magnesium during molecular beam epitaxy (MBE) has been studied. In the nitrogen rich regime of MBE growth, GaN films are known to grow with rough morphology. We observe on GaN(0001) that small doses of $\mathrm{Mg}$ act as a surfactant, smoothing out this roughness. An interpretation of this surfactant behavior is given in terms of electron counting arguments for the surface reconstructions. Previously, we have reported that larger doses of $\mathrm{Mg}$ lead to inversion of the Ga-polar GaN film to produce N-polar GaN. Several Mg-related reconstructions of the resulting $\mathrm{GaN}(000 \overline{1})$ surface are reported.
\end{abstract}

\section{INTRODUCTION}

Surfactant mediated film growth has been an important area of work for both film growers and surface scientists. For the epitaxy of thin films on lattice-mismatched substrates, surfactants provide a reduction in surface energy which balances the strain energy in the film, thereby enabling two-dimensional growth (without incorporation of the surfactant). Thus a potential application of surfactants is in the initial nucleation of $\mathrm{GaN}$ on mismatched substrates such as $\mathrm{SiC}$ or sapphire. Once nucleation has occurred and the film has grown thicker, surfactants may improve surface morphology of the film by affecting the growth mode or growth rates of different lattice planes, again favoring a more two-dimensional film. For high quality GaN devices, it is important to have both excellent bulk quality as well as a flat surface morphology. From this point of view, it is very important to explore the area of surfactants for GaN film growth. Various chemical species have been found to exhibit surfactant type effects during GaN growth, including magnesium (as seen in the growth rate of $\mathrm{GaN}$ on different crystallographic planes when it is doped with $\mathrm{Mg}$ ) [1], arsenic [2], and indium [3].

In this work, we show evidence for a surfactant effect due to $\mathrm{Mg}$ during $\mathrm{GaN}$ growth. As has been noticed by several MBE growers, GaN growth in Ga-rich conditions produces smooth surfaces indicative of two-dimensional growth. But growth under N-rich conditions leads to three-dimensional, rough surfaces, for both Ga- and N-polar films [4,5]. Under N-rich conditions, we see that exposure of these Ga-polar rough films to sub-monolayer quantities of $\mathrm{Mg}$ leads to a smoothing of the film. An interpretation of this surfactant behavior of the $\mathrm{Mg}$ is given in terms of electron counting arguments for the surface reconstructions.

\section{EXPERIMENT}

The films are grown in a molecular beam epitaxy (MBE) chamber with base pressure less than $1 \times 10^{-10}$ Torr, using Si-polar $6 \mathrm{H}-\mathrm{SiC}(0001)$ substrates. The substrates are prepared 
ex situ by hydrogen etching [6]. The surface oxide is removed by Si pre-deposition and desorption [7] till a $\sqrt{ } 3 \times \sqrt{ } 3 \mathrm{R}-30^{\circ}$ reconstructed surface is obtained. Immediately after this, the substrate is brought to growth temperature and growth is initiated. Growth temperatures are in the range $600-700^{\circ} \mathrm{C}$. Growth conditions are usually Ga-rich, for which we observe a streaky $1 \times 1$ RHEED pattern during growth. However, for our studies of the surfactant effect of $\mathrm{Mg}$, we have grown films in the $\mathrm{N}$-rich regime before $\mathrm{Mg}$ exposure. The hallmark of the N-rich regime is a spotty, bright RHEED pattern. Ga and $\mathrm{Mg}$ fluxes are produced by effusion cells, while an RF-plasma is used to excite $\mathrm{N}_{2}$ molecules thereby facilitating reaction. $\mathrm{Mg}$ exposure is done during growth and is seen not to have any effect when performed during growth interrupts. This may have to do with the short residence times for $\mathrm{Mg}$ atoms on the GaN surface at the growth temperature. During growth, atoms on the surface are mobile enough for $\mathrm{Mg}$ to get incorporated in the crystal surface immediately upon their arrival, whereas even with prolonged exposures during interrupts little $\mathrm{Mg}$ sticks to the surface. Following growth, surfaces are characterized using in situ scanning tunneling microscopy (STM) and ex situ atomic force microscopy (AFM), as described elsewhere [8].

\section{RESULTS}

\section{Surfactant Behavior of $\mathrm{Mg}$}

GaN films grown in the Ga-rich regime show a smooth two-dimensional (2-D) morphology. In Fig. 1(a), a RHEED pattern and AFM image obtained for a film grown under these conditions are displayed. The RHEED pattern is dim and streaky, since the growth is 2-D and at all times the surface is covered with excess Ga (at least 2 disordered ML) [8]. The AFM image displays deep pits and trenches (> $150 \AA$ Aeep) which are characteristic of the columnar growth in the MBE process. In between these pits, however, the film shows a flat, 2-D morphology with atomic steps faintly visible. As the Ga flux becomes smaller, the RHEED pattern becomes brighter and eventually in the N-rich region of growth, the RHEED pattern begins to get spotty as shown in the inset in Fig. 1(b). The morphology of the film in the N-rich regime is shown in Fig. 1(b), and the roughening is seen as the granular appearance of the film between the deep pits.

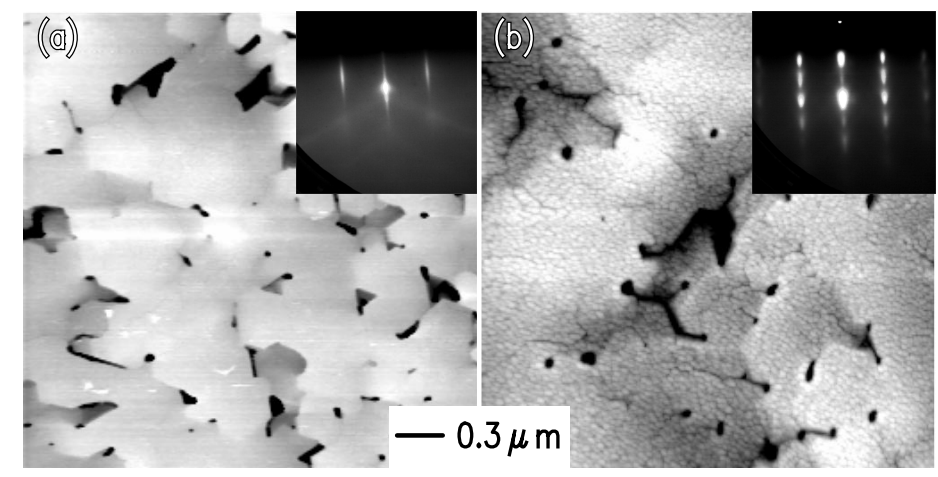

Figure 1 ( a) AFM image of a film grown under Ga-rich conditions, (b) grown under N-rich conditions. In the insets we see corresponding RHEED patterns in the (11 $\overline{2})$ azimuth. 
During growth, films are briefly exposed to a Mg flux. A surfactant effect of $\mathrm{Mg}$ is seen on these Ga-polar films in the N-rich regime. Exposing the growth surface to as little as $0.2 \mathrm{ML}$ of $\mathrm{Mg}$ under Ga-poor conditions leads to a reversal of the RHEED pattern from spotty to streaky. Also, when the growth is made very N-rich by reducing the Ga flux to about one half of that at the transition point, exposure to $\mathrm{Mg}$ often produces a streaky $2 \times 2$ pattern. At typical sample temperatures during growth $\left(625^{\circ} \mathrm{C}\right.$ for the sample shown below), the sticking coefficient of $\mathrm{Mg}$ on the $\mathrm{GaN}$ surface is expected to be rather low, therefore the $0.2 \mathrm{ML}$ estimate we make from room temperature calibrations of the $\mathrm{Mg}$ source is an upper limit on the amount required to cause this surfactant effect. Figure 2 shows AFM images which present a sequence of surface morphologies during the smoothing of the surface. Figure 2(a) shows a rough film similar to the one in Fig. 1(b) grown in the N-rich regime. An exposure of about $0.2 \mathrm{ML}$ of $\mathrm{Mg}$ leads to a film which shows areas such as those in Fig. 2(b). As indicated by the arrows, regions of 2-D growth start to nucleate on the rough film, where we can see atomic steps again. At about $0.7 \mathrm{ML} \mathrm{Mg}$ exposure, the surface is mostly smoothed out and the film shows morphology as in Fig. 2(c) and (d). The morphology of Fig. 2(d) is indistinguishable from that of Fig. 1(a), but small areas of roughness are still seen in Fig. 2(c). Closer inspection reveals that these patches of roughness follow the directions of steps, suggesting that the smoothing is a step-flow process.

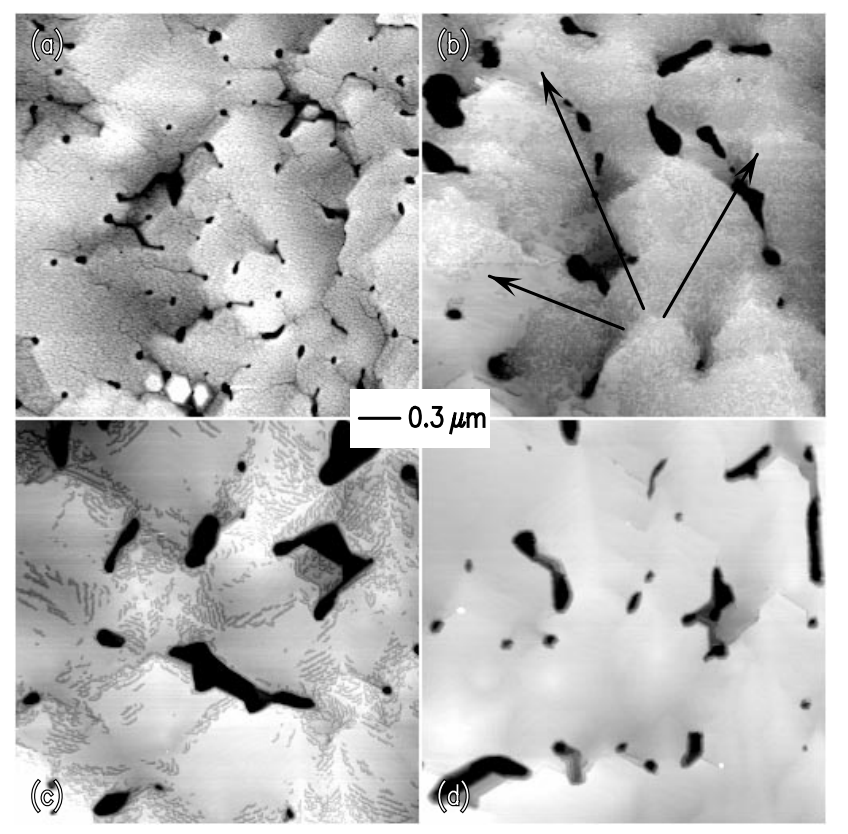

Figure 2 AFM images showing the surfactant effect of Mg: (a) GaN film grown in N-rich regime without $\mathrm{Mg},(b)$ and $(c)$ intermediate stages of the smoothing process, $(d)$ complete smoothing of surface as a result of $\mathrm{Mg}$ exposure. The arrows in (b) point to smooth areas beginning to appear interspersed amidst the rough regions. 
From our RHEED and AFM observations, it is apparent that Mg has a surfactant effect under Ga deficient growth conditions. The surfactant effect is seen to persist for some time after the exposure of $\mathrm{Mg}$, confirming previous observations that $\mathrm{Mg}$ segregates to the surface during GaN growth [9]. However, extensive use of this surfactant effect is made difficult by the Mg-induced crystal polarity inversion reported previously [10], in which exposure of the surface to $>1 \mathrm{ML}$ of $\mathrm{Mg}$ is found to change the film polarity from Ga-polar to N-polar.

\section{Mg-derived reconstructions of $\mathrm{GaN}(000 \overline{1})$}

We have also observed several Mg-induced low temperature reconstructions on the $\mathrm{GaN}$ surface after $\mathrm{Mg}$ has led to inversion, i.e. on the (0001) face. These are obtained by exposing the $\mathrm{GaN}$ surface to a $\mathrm{Mg}$ flux while holding the substrate at a temperature around $250^{\circ} \mathrm{C}$. Upon deposition of about $0.04-0.08 \mathrm{ML}$ of $\mathrm{Mg}$, we see a $5 \times 5$ reconstruction. With the addition of more $\mathrm{Mg}$, we see the appearance of $4 \times 4,3 \times 3$ and $6 \times 6$ RHEED patterns. These patterns may be observed in the reverse order upon heating the $6 \times 6$ at around $400^{\circ} \mathrm{C}$, and prolonged heating at this temperature leads to the disappearance of these patterns and the reappearance of the $1 \times 1$ usually seen on the $\mathrm{N}$-polar face. Thus it is clear that these reconstructions arise from $\mathrm{Mg}$ atoms that are weakly bound to the surface. Figure 3 shows STM images corresponding to the $5 \times 5$ reconstruction. The structure is composed of a hexagonal array of corrugation maxima (probably adatoms), arranged in a $5 \times 5$ pattern. Some regions of the surface were covered with additional adsorbates (possibly excess $\mathrm{Mg}$ or $\mathrm{Ga}$ ), forming the bright regions in Fig. 3(a). At this time we do not have detailed models for the structure of these various reconstructions, although it seems likely that they can be constructed by substituting $\mathrm{Mg}$ atoms for $\mathrm{Ga}$ atoms in the $\mathrm{GaN}(000 \overline{1})$ adatom-on-adlayer structures [11]. For example, the $5 \times 5$ structure could correspond to having $2 \mathrm{Mg}$ atoms $/$ cell in adatom sites above the $1 \times 1$ adlayer.
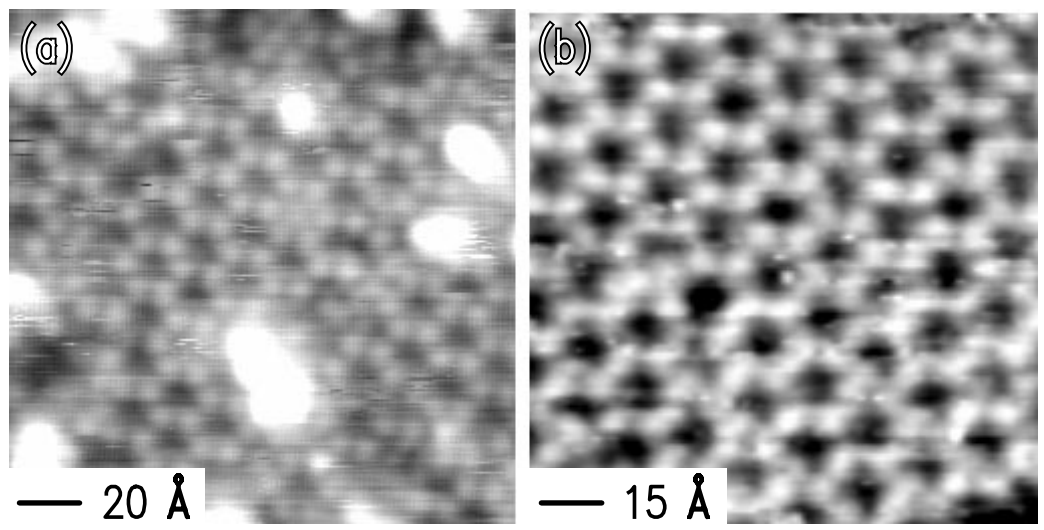

Figure 3 STM images of $5 \times 5$ Mg-induced reconstruction on the $N$-polar GaN surface, acquired at sample voltages of $(a)+2.0 \mathrm{~V}$ and $(b)+2.5 \mathrm{~V}$, and at a constant current of 0.1 $n A$. Grey-scale ranges are 1.4 and $0.5 \AA$ for $(a)$ and $(b)$ respectively. 


\section{DISCUSSION}

As discussed above, the presence of small amounts of $\mathrm{Mg}$ on the $\mathrm{GaN}(0001)$ surface, growing under $\mathrm{N}$-rich conditions, can lead to a conversion of the nominally rough surface (spotty RHEED pattern) to a smooth surface (streaky RHEED pattern). Concomitantly, a $2 \times 2$ RHEED pattern can be seen on the surface. Although we have not studied this $2 \times 2$ structure in detail (e.g. using STM), we can discuss its structure based on recent theoretical results [12]. Considering a variety of $2 \times 2$ arrangements, Bungaro et al. find that $1 / 4 \mathrm{ML}$ of $\mathrm{Mg}$ substituting for $\mathrm{Ga}$ on the surface is energetically the most stable configuration (except for very Ga-rich conditions, which are not of concern here). In terms of electron counting, this surface would have 2 electrons available to fill dangling-bond derived surface states, so that a surface gap could develop and we would anticipate relatively low diffusion barriers compared to the Ga-terminated (0001) surface.

Another model for the Mg-induced 2×2 structure is possible, containing 3/4 ML of $\mathrm{Mg}$ substituting for surface Ga atoms. In this case, all dangling bonds on the surface would be completely empty, and again we would anticipate relatively low diffusion barriers. Based on total energy computations of the type described elsewhere [10], we find that this 3/4 ML model will be energetically favorable for Mg-rich and/or Ga-poor conditions. Explicitly, the energy difference between the $1 / 4$ and 3/4 ML models may be expressed as

$$
\Delta E(e V / 2 \times 2)=0.05 \mathrm{eV}-2(\Delta \mu G a-\Delta \mu M g)
$$

where $\Delta \mu \mathrm{Ga}$ and $\Delta \mu \mathrm{Mg}$ are the chemical potentials measured relative to their bulk values. A positive energy means that the 3/4 ML structure is preferred over the 1/4 ML structure. If $\Delta \mu \mathrm{Ga}=\Delta \mu \mathrm{Mg}$ (say, both are zero) then the 3/4 ML structure is preferred. In extreme $\mathrm{N}$-rich conditions, we could have $\Delta \mu G a \approx-1.0 \mathrm{eV}$. Under such conditions it is possible to stabilize the 3/4 ML structure for values of $\Delta \mu M g>-1 \mathrm{eV}$. In our experiments we find that $0.2 \mathrm{ML}$ of $\mathrm{Mg}$ can lead to the surfactant behavior, which would argue in favor of the $1 / 4$ ML model as correctly describing the surface structure. On the other hand, the smooth areas of that surface extend over only a small portion of the total surface area (Fig. 2(b)), so that the 3/4 ML model may also be consistent with the data. Additional experimental study of the surface structure is required to more definitively determine the structure.

Thus, we believe that the presence of the Mg allows the surface to satisfy electron counting (at least for the 3/4 ML model), thereby leading to completely filled or completely empty surface state bands. Qualitatively, one expects such a surface to be non-reactive, and have relatively low diffusion barriers compared to the bare GaN surface [13]. Hence, a surfactant effect (smooth, 2-D growth) could be expected, in agreement with the experimental observations. A similar situation is believed to occur on arsenic exposed surfaces. In that case, near the critical Ga flux for the transition from smooth to rough morphology we find, in the presence of arsenic, an intense $2 \times 2$ streaky RHEED pattern, indicative of smooth morphology [14]. This pattern persists for a range of Ga fluxes both above and below the critical flux, thus demonstrating a surfactant behavior of the arsenic. Our interpretation of the $2 \times 2$ structure is that it arises from arsenic adatoms on the Ga-terminated surface (we believe that a number of groups have mistakenly identified this structure as arising from $\mathrm{Ga}$ adatoms, since the surface arsenic in their cases unintentionally arises from background arsenic contamination in their vacuum systems [14-16]). The $2 \times 2$ As-adatom surface satisfies electron counting, so again we expect relatively low diffusion barriers in that case. 


\section{SUMMARY}

In summary, we have grown GaN films on hydrogen-etched $6 \mathrm{H}-\mathrm{SiC}(0001)$ by MBE at growth temperatures of $600-700^{\circ} \mathrm{C}$ with brief exposures to $\mathrm{Mg}$. The $\mathrm{Mg}$ exposures are seen to have a surfactant effect at low coverage, and at high coverage to invert the Ga-polar $\mathrm{GaN}$ film to a N-polar film. An interpretation of the surfactant effect is given in terms of electron counting arguments, and this effect is compared with that observed for arsenic overlayers. We have also observed low temperature reconstructions on the inverted surface, which may help in the understanding of $\mathrm{Mg}$ incorporation in $\mathrm{N}$-polar GaN.

\section{ACKNOWLEDGEMENTS}

This work was supported by the Office of Naval Research, grant N00014-96-1-0214 monitored by Dr. Colin Wood, and by the National Science Foundation, grant DMR9615647.

\section{REFERENCES}

[1] B. Beaumont, S. Haffouz and P. Gibart, Appl. Phys. Lett. 72, 921 (1998).

[2] Y. Zhao, F. Deng, S. S. Lau, and C. W. Tu, J. Vac. Sci. Technol. 16, 1297 (1998).

[3] F. Widmann, B. Daudin, G. Feuillet, N. Pelekanos, and J. L. Rouvière, Appl. Phys. Lett. 73, 2642 (1998).

[4] E. J. Tarsa, B. Heying, X. H. Wu, P. Fini, S. P. DenBaars, and J. S. Speck, J. Appl. Phys. 82, 5472 (1997).

[5] A. R. Smith, V. Ramachandran, R. M. Feenstra, D. W. Greve, A. Ptak, T. H. Myers, W. L. Sarney, L. Salamanca-Riba, M.-S. Shin and M. Skowronski, MRS Internet J. Nitride Semicond. Res. 3, 12(1998).

[6] V. Ramachandran, M. F. Brady, A. R. Smith, R. M. Feenstra and D. W. Greve, J. Electron. Mater. 27, 308 (1998).

[7] V. Ramachandran, A. R. Smith, R. M. Feenstra and D. W. Greve, J. Vac. Sci. Technol. A 17, 1289 (1999).

[8] A. R. Smith, R. M. Feenstra, D. W. Greve, M. S. Shin, M. Skowronski, J. Neugebauer, J. E. Northrup, J. Vac. Sci. Technol. B 16, 2242 (1998).

[9] T. S. Cheng, C. T. Foxon, N. J. Jeffs, D. J. Dewsnip, L. Flannery, J. W. Orton, S. V. Novikov, B. Ya Ber and Yu A. Kudriavtsev, MRS Internet J. Nitride Semicon. Res. 2, 13 (1997).

[10] V. Ramachandran, R. M. Feenstra, W. L. Sarney, L. Salamanca-Riba, J. E. Northrup, L. T. Romano, and D. W. Greve, Appl. Phys. Lett. 75, 808 (1999).

[11] A. R. Smith, R. M. Feenstra, D. W. Greve, J. Neugebauer, and J. Northrup, Phys. Rev. Lett. 79, 3934 (1997).

[12] C. Bungaro, K. Rapcewicz, and J. Bernholc, Phys. Rev. B 59, 9771 (1999).

[13] T. Zywietz, J. Neugebauer, and M. Scheffler, Appl. Phys. Lett. 73, 487 (1998).

[14] V. Ramachandran, C. D. Lee, R. M. Feenstra, A. R. Smith, J. E. Northrup, and D. W. Greve, J. Cryst. Growth 126, to appear.

[15] K. Iwata, H. Asahi, S. J. Yu, K. Asami, H. Fujita, M. Fushida, and S. Gonda, Jpn. J. Appl. Phys. 35, L289 (1996).

[16] Q. K. Xue, Q. Z. Xue, R. Z. Bakhtizin, Y. Hasegawa, I. S. T. Tsong, T. Sakurai, and T. Ohno, Phys. Rev. Lett. 82, 3074 (1999). 